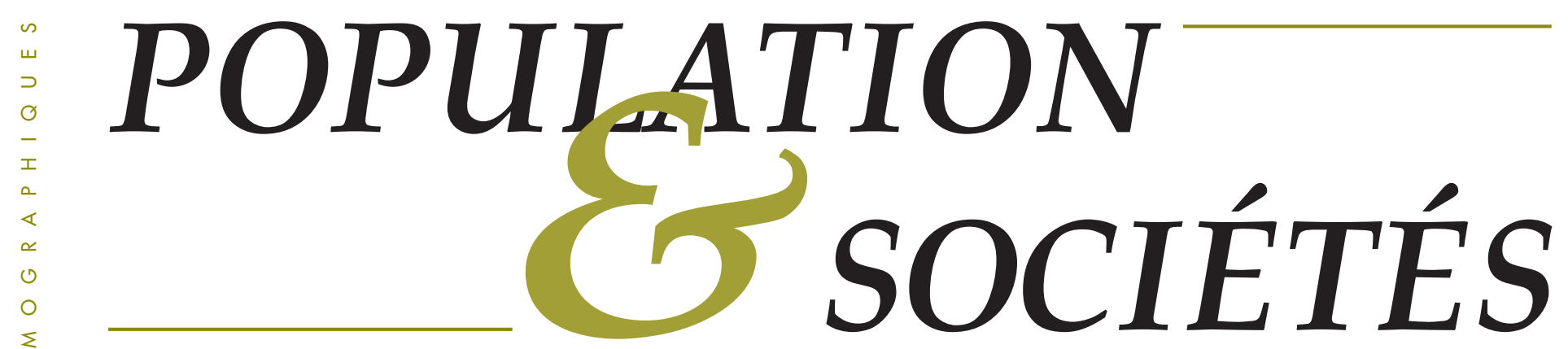

\title{
Comment les personnes dépendantes seront-elles entourées en 2030 ? Projections européennes
}

\author{
Joëlle Gaymu* et l'équipe FELICIE**
}

\begin{abstract}
Le vieillissement démographique fait craindre une augmentation considérable du nombre de personnes âgées souffrant d'incapacités et ayant besoin d'aide. Combien y aura-t-il de personnes dans cette situation demain? Comment vivront-elles? Auront-elles un conjoint ou un enfant qui puisse s'occuper d'elles? Pour connaître la situation en Europe, une équipe de chercheurs a calculé des projections de population âgée dépendante jusqu'en 2030 en distinguant différentes situations familiales. Joëlle Gaymu, qui a coordonné cette équipe, nous livre ici les principaux résultats de l'étude.
\end{abstract}

$\mathrm{D}$ ici à 2030, partout en Europe, la population âgée de 75 ans ou plus augmentera fortement. Or, même si la majorité des personnes âgées vieillissent en bonne santé, le risque de dépendance physique et psychique progresse avec l'âge. Une aide régulière devient alors, généralement, indispensable et, dans ce cas, les premiers pourvoyeurs sont le conjoint et les enfants: leur absence ou leur défaillance augmente, ipso facto, le besoin d'aide professionnelle. Mais les Européens âgés en situation de dépendance pourront-ils compter demain sur des aidants familiaux plus qu'aujourd'hui? Une étude soutenue par la Commission européenne a exploré les évolutions probables au cours des deux ou trois prochaines décennies (voir encadré).

\section{Demain avoir un conjoint ou un enfant sera plus fréquent}

En l'an 2000, en Europe, les chances d'avoir un conjoint auprès de soi lorsqu'on a 75 ans ou plus et qu'on est en situation d'incapacité sont bien plus élevées pour les hommes que pour les femmes: $60 \%$ contre $19 \%$. La majeure partie des femmes $(62 \%)$ ne peuvent compter que sur l'aide éventuelle d'un enfant. Plus souvent que les hommes (19\% contre $12 \%)$, elles se retrouvent sans

\footnotetext{
- Institut national d'études démographiques

** Future Elderly Living Conditions in Europe
}

conjoint ni enfant. Enfin, pour les uns comme pour les autres, il est rare de n'avoir que le conjoint comme recours possible (figure 1).

À l'avenir, l'entourage familial des personnes âgées est amené à changer en raison de deux tendances de fond (voir encadré) : la baisse du veuvage et la proportion croissante de divorcés [1]. Mais ces évolutions devraient varier selon le sexe et l'âge.

Chez les femmes, la montée des divorces sera plus que contrebalancée par le recul très marqué du veuvage dû à la baisse de la mortalité et au rapprochement des espérances de vie entre hommes et femmes. Elles pourront ainsi compter plus fréquemment sur la présence d'un partenaire pour faire face à leur dépendance et ceci dans tous les pays. La tendance est particulièrement soutenue chez les Européennes de 85 ans et plus: à ces âges, la présence d'un conjoint sera, quasiment partout, trois fois plus fréquente en 2030 qu'en 2000 ( $22 \%$ contre $9 \%$ ).

Du côté des hommes de 85 ans et plus, la baisse du veuvage jouera dans le même sens et leur assurera plus souvent le soutien d'un partenaire en cas de dépendance ( $53 \%$ en 2030 contre $42 \%$ en 2000). Ceux de 75 84 ans seront également moins souvent veufs (13\% en 2030 contre $21 \%$ en 2000) mais aussi plus fréquemment divorcés (13\% contre $3 \%)$. À ces âges, il y aura donc moins d'hommes en couple. Cette tendance s'observera 
dans la plupart des pays, sauf au Royaume-Uni, en Italie, au Portugal et en République Tchèque, où leurs chances de vivre à deux resteront inchangées.

Avec l'arrivée à l'âge de 75 ans des générations nées dans l'entre deux guerres et ayant eu leurs enfants pendant le baby-boom, la proportion de personnes sans enfant survivant diminuera ou restera stable, sauf chez les hommes de 75-84 ans en Allemagne, en Finlande et aux Pays-Bas. À ces exceptions près, l'absence de tout soutien familial en cas de dépendance sera moins fréquente. Actuellement, le risque est au plus haut chez les femmes de 85 ans et plus ( $23 \%$ en 2000$)$; il reculera à $15 \%$ en 2030 .

Hormis les hommes de 75 à 84 ans, les Européens âgés en situation de dépendance verront leur environnement familial s'étoffer, sans qu'on sache dans quelle mesure cet entourage sera réellement désireux et capable de faire face à la dépendance du conjoint, des parents ou des beaux-parents. Par ailleurs, la forte croissance attendue de la population âgée risque d'entraîner, en nombres absolus, une augmentation de la population sans famille, même si, proportionnellement, elle doit reculer. Or l'évolution des effectifs est un facteur capital à prendre en compte pour définir la politique de prise en charge de la dépendance et des futurs services.

\section{La population dépendante sans aidants familiaux potentiels pourrait rester stable}

Si l'état de santé reste identique, d'ici à 2030, la population des Européens dépendants âgés de 75 ans ou plus pourrait augmenter de $72 \%$, et même quasiment doubler en Finlande, en République tchèque et aux Pays-Bas. Ailleurs, les taux de progression s'échelonneraient de moins de $50 \%$ (Portugal) à $80 \%$ (France). Cette hiérarchie reflète en grande partie l'intensité inégale du baby-boom en Europe [2]. Avec l'hypothèse (ici faite) d'une amélioration de l'état de santé (voir encadré), les taux ne varieraient plus que de $74 \%$ (Pays-Bas) à 25\% (Portugal), pour une valeur moyenne de $41 \%$.

La fraction de cette population qui sera sans conjoint ni enfant va progresser beaucoup plus faiblement en comparaison, sauf en Finlande. Dans l'hypothèse d'une stabilité de l'état de santé, l'effectif de personnes cumulant ces deux situations augmentera seulement de $25 \%$ d'ici à 2030 .

Cela signifie qu'à l'avenir, les personnes qui n'auront d'autre recours que les services professionnels -y compris l'entrée en institution - verront leurs effectifs croître beaucoup moins vite que les personnes ayant un entourage familial proche. En outre, une amélioration de l'état de santé pourrait changer considérablement l'amplitude de la croissance des besoins d'assistance. Dans le scénario envisagé ici, non
Figure 1 - Situation familiale des personnes âgées dépendantes en Europe en 2000 et 2030

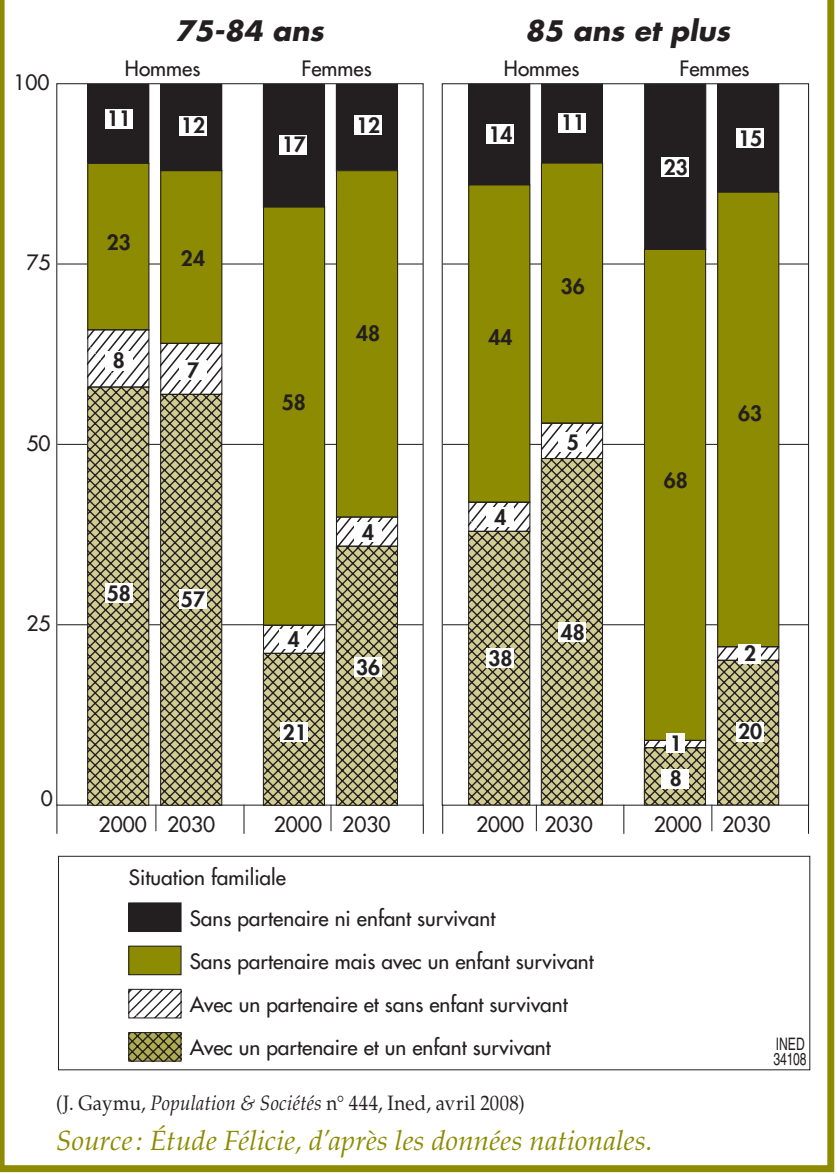


Figure 3 - Évolution de la population âgée dépendante en Europe d'ici 2030*
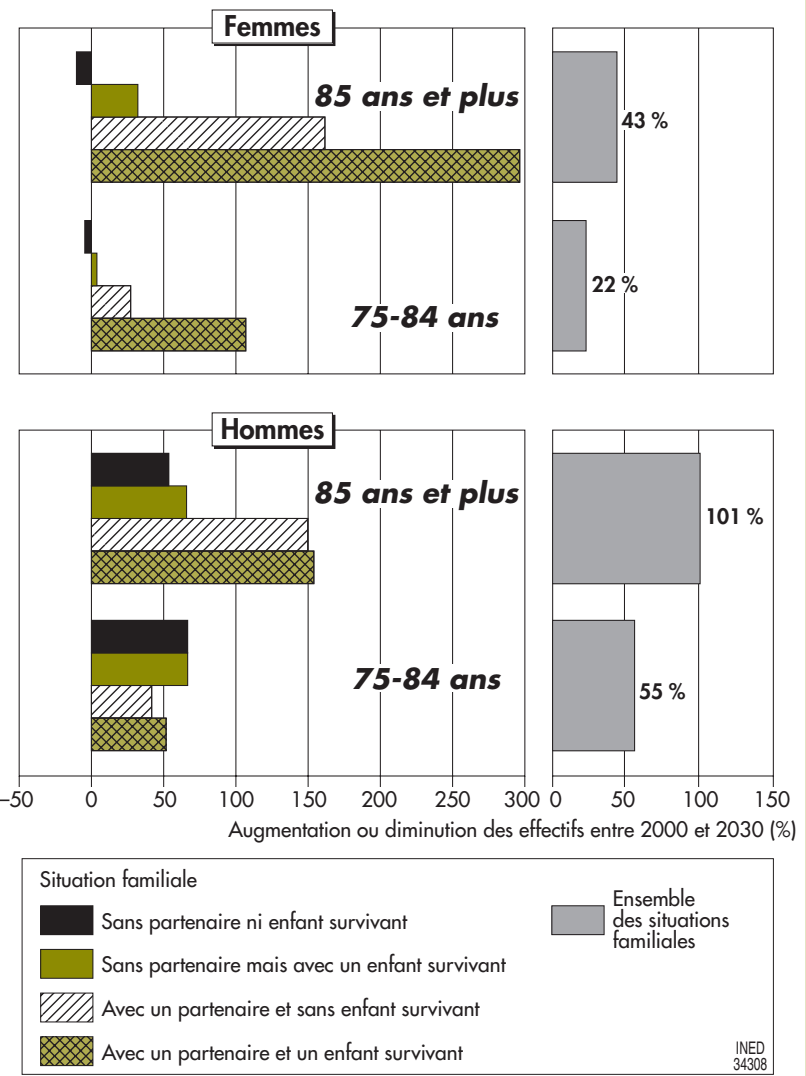

(J. Gaymu, Population \& Sociétés n ${ }^{\circ} 444$, Ined, avril 2008)

Source: Étude Félicie, d'après les données nationales.

* scénario avec amélioration de l'état de santé.

seulement le taux de croissance de la population dépendante serait quasiment divisé par 2 (reculant de $72 \%$ à $41 \%$ ) mais la population n'ayant plus d'aidant familial diminuerait ou resterait stable partout, sauf aux Pays-Bas et en Finlande.

\section{Et celle ayant à la fois conjoint et enfant survivant va augmenter le plus fortement}

Si le fait d'avoir un partenaire ou un enfant survivant vous rend a priori moins vulnérable aux situations de dépendance, les conditions de vie peuvent devenir critiques quand le conjoint est en mauvaise santé ou que l'enfant se dérobe. Ne pouvoir compter que sur l'aide d'un enfant est la situation type des femmes âgées dépendantes. Or la progression de cette population sera soutenue (de $47 \%$ ou $22 \%$ en moyenne selon le scénario envisagé) mais plus faible que celle de l'ensemble. Le nombre de personnes ayant un conjoint comme seul soutien croîtra plus fortement, cette configuration familiale restant, toutefois, rare. Finalement, d'ici à 2030, la plus forte hausse concernera la population ayant à la fois un conjoint et un enfant. Si l'état de santé reste stable, elle augmentera de $65 \%$ (au Portugal) à plus de $200 \%$ (en Finlande et en République tchèque) et de $145 \%$ en moyenne. S'il s'améliore, la progression sera seulement de $100 \%$, s'échelonnant de 39\% (au Portugal) à $138 \%$ (en Finlande) (figure 2). C'est dire que la lourdeur de la prise en charge de la dépendance ne
Encadré

\section{Le programme de recherche FELICIE * (www.felicie.org)}

Le programme de recherche $\mathrm{FELICIE}{ }^{*}$ vise à prévoir les contextes familiaux des personnes âgées de demain dans un échantillon de neuf pays européens (Allemagne, Belgique, Finlande, France, Italie, Pays-Bas, Portugal, République tchèque et Royaume Uni).

Un premier jeu de projections démographiques a été calculé distinguant l'état matrimonial des individus et leur modification au fil du temps et des générations. Ces projections ont servi de base ensuite pour calculer différentes projections dérivées selon deux scénarios d'évolution de l'état de santé. Dans le premier, les taux $d^{\prime}$ incapacité ${ }^{* *}$ par âge et état matrimonial ont été maintenus constants. Le second scénario fait l'hypothèse que toutes les années gagnées en espérance de vie sont des années en bonne santé. Les taux d'incapacités diminuent alors à chaque âge de l'ordre de $20 \%$ d'ici à 2030. Certaines évolutions comme l'élévation du niveau d'instruction de la population âgée, la moindre pénibilité des professions, l'amélioration des comportements de prévention, sont plutôt en faveur du second scénario [5]. Dans un troisième temps, les différentes sous-populations ainsi obtenues (par âge, sexe, état matrimonial et état de santé) ont été réparties selon l'existence ou non d'un enfant survivant.

Ces projections n'intègrent pas d'éventuels changements en matière d'obligations entre parents et enfants, de désir d'indépendance résidentielle des générations, de normes sociales ou encore $d^{\prime}$ orientation politique en matière de prise en charge de la dépendance. Leur but est de montrer comment les changements de certaines caractéristiques des aînés -en grande partie déjà inscrits dans I'histoire passée des générations - vont transformer leur contexte familial tant dans leur structure que dans leurs effectifs.

\section{Un programme de recherche européen}

Le programme de recherche FELICIE* est financé par la Commission européenne. Il rassemble près $d^{\prime}$ une trentaine de chercheurs appartenant à une dizaine d'instituts de recherches en Europe: G. Doblhammer et W. Apt (MPIDF) pour l'Allemagne; M. Poulain et L. Dal (GEDAP) pour la Belgique; P. Martikainen et E. Nihtilä (Université d'Helsinki) pour la Finlande; C. Delbes, A. Desesquelles, P. Festy, J. Gaymu, S. Pennec et S. Springer (Institut national d'études démographiques) pour la France; G. De Santis, C. Seghieri et M. L. Tanturri (Université de Florence) pour I'Italie; G.Beets, H. Cruisen, P Ekamper, E. Van Imhoff et L. Van Vissem (NIDI) pour les Pays-Bas; A. Fernandes, T. Veiga et F. de Castro Henriques (Université de Lisbonne) pour le Portugal ; J. Rychtarikova (Université Charles de Prague) pour la République Tchèque; E. Grundy et C. Tomassini (LSHTM) et S. Kalogirou et M. Murphy (LSE) pour le Royaume-Uni.

* Future Elderly Living Conditions in Europe. Pour en savoir plus: Les cahiers de l'Ined, $n^{\circ} 162$, Paris, 250 p. (à paraître, 2008).

** La dépendance est définie à partir de la question utilisée dans l'Europanel «Etes-vous gêné dans vos activités quotidiennes par des problèmes de santé physique ou psychique, des maladies ou handicaps? ». Les personnes ayant répondu "sévèrement» ont été retenues pour définir la population dépendante. La population institutionnalisée a ensuite été réintégrée dans la population dépendante.

dépend pas seulement de l'augmentation du nombre des personnes âgées.

\section{Le profil des aînés dépendants et des aidants familiaux va changer}

Un élément-clef à prendre en compte est la composition de la population dépendante par sexe et âge. En premier lieu, elle vieillira inexorablement. S'il y a amélioration de l'état de santé, côté hommes, les effectifs des 85 ans ou plus doubleront alors que les 75-84 ans augmenteront seulement de $55 \%$. Même décalage chez les femmes: 


\section{Pierre Longone \\ (1911-2008)}

Pierre Longone, fondateur de Population et sociétés, est mort le 10 mars 2008 à l'âge de 96 ans. Il avait lancé le premier numéro du bulletin en mars 1968, il y a 40 ans. Il s'occupait auparavant de la rédaction du bulletin mensuel du comte de Paris, qui commentait l'actualité politique, économique et sociale à destination des parlementaires, des journalistes et des dirigeants d'entreprise. Il quitte le comte de Paris en 1968 pour être recruté comme chef du service des éditions de l'Institut national d'études démographiques (Ined). Il vient avec la formule du bulletin mensuel du comte de Paris et le fichier des abonnés. Telle est l'origine inattendue de la formule du bulletin d'information qui, à partir de l'Ined, se diffusera largement dans toute l'administration française. Pierre Longone rédigeait lui-même la plupart des numéros de Population et sociétés. Il s'acquittera de cette mission jusqu'à sa retraite en 1977.

Quarante ans après son lancement, Population et sociétés est toujours là et continue d'informer un vaste public sur les évolutions démographiques et leurs ressorts. Le bulletin est dorénavant publié en deux langues: français et anglais, et est accessible gratuitement en ligne: www.ined.fr. Son format a peu changé en 40 ans, la formule restant celle à succès inventée par Pierre Longone il y a 40 ans.

F. Héran et G. Pison

$43 \%$ contre $22 \%$ (figure 3). Il s'ensuivra des besoins accrus d'aide professionnelle, puisque avec l'âge, les incapacités deviennent plus sévères et l'aide des membres de la famille (eux-mêmes plus âgés) moins efficace.

En outre, les femmes de 85 ans ou plus vivant en couple verront leur nombre multiplié par un facteur allant de 2,5 (Royaume-Uni, France et Italie) à 5 (Allemagne et Finlande). De plus en plus d'hommes seront donc confrontés à la dépendance de leur partenaire, et ceci dans tous les pays: au sein de la population dépendante de 85 ans et plus, la part des femmes vivant en couple pourrait passer de $7 \%$ à $16 \%$ d'ici à 2030 . Or, les hommes d'aujourd'hui ont plus de difficultés à assumer la dépendance de leur épouse que l'inverse: ils ont plus souvent recours à des aides professionnelles [3] ou placent plus fréquemment leur conjointe en institution [4]. Réciproquement, une fraction croissante de femmes âgées de 85 ans et plus devront prendre en charge leur époux dépendant. Au total, les personnes dépendantes

\section{RÉFÉRENCES}

[1] Kalogirou S., Murphy M. - «Marital status of people aged 75 and over in nine EU countries in the period 2000$2030 »$, European Journal of Ageing, 3, 2006, p. 74-81.

[2] Monnier A. - «Le baby-boom: suite et fin», Population $\mathcal{E}$ Sociétés, n 431, février 2007.

[3] MARTEl L., LÉGARÉ J. - «Avec ou sans famille proche à la vieillesse: une description du réseau de soutien informel des personnes âgées selon la présence du conjoint et des enfants», Cahiers québécois de démographie, volume 30, n 1, 2001, p. 89-114.

[4] Delbès C., Gaymu J., Springer S. - «Les femmes vieillissent seules, les hommes à deux. Un bilan européen ", Population $\mathcal{E}$ Sociétés, $\mathrm{n}^{\circ}$ 419, janvier 2006.

[5] Robine J.-M., Romieu I., Michel J.P. - «Trends in health expectancies", in J.-M. Robine, C. Jagger, C.-D. Mathers, E.-M. CRImmins and R.-M. Suzman (Eds.), Determining health expectancies, Wiley, Chichester, West Sussex, 2003, p. 75-101. vivront en couple dans $31 \%$ des cas en 2030, contre $16 \%$ en 2000. Or, actuellement, le conjoint présente également des problèmes de santé dans environ la moitié des cas. Il est à prévoir que la population dépendante sera plus souvent composée de couples dont les deux membres souffriront d'incapacités.

Dans la majorité des cas, le surplus de population souffrant d'incapacités dans les trois décennies à venir sera composé de personnes ayant potentiellement des aidants familiaux. Toutefois, l'incertitude reste de mise sur leur mobilisation réelle. En outre, la croissance de la part des personnes dépendantes très âgées, la masculinisation des aidants conjugaux et la survie plus fréquente de couples dont les deux membres seront dépendants sont autant de facteurs qui feront grossir la population demandeuse d'une aide d'ordre professionnel. À l'avenir, plus encore qu'aujourd'hui, les politiques de prise en charge de la dépendance devront envisager en priorité l'aide aux aidants.

\section{RÉSUMÉ}

La population de personnes dépendantes âgées de 75 ans ou plus devrait augmenter de plus de $70 \%$ en Europe d'ici 2030 dans les conditions de santé d'aujourd'hui. Dans le même temps, la part dans cette population des personnes sans conjoint ni enfant devrait diminuer au profit des personnes ayant au moins un parent (enfant ou conjoint) pouvant éventuellement les aider. Les situations resteront contrastées entre les hommes et les femmes en situation d'incapacité, les chances pour les premiers d'avoir un conjoint auprès d'eux étant bien plus élevées que pour elles. La croissance de la part des personnes dépendantes très âgées, la masculinisation des aidants conjugaux et la survie plus fréquente de couples dont les deux membres seront dépendants feront grossir la population demandeuse d'une aide d'ordre professionnel. 